\title{
Analytical and Numerical Investigation of Transient Gas Blow down
}

\author{
Hameed Balassim Mahood, Farhan Lafta Rasheed \& Abdulsattar K. Abbas \\ Ministry of Sciences and Technology, Baghdad, Iraq
}

Received: August 8, 2011

Accepted: August 26, 2011

doi:10.5539/mas.v5n5p64

\begin{abstract}
An analytical and numerical solution for unsteady discharge of gas through blow down stack open to atmosphere based upon the volume model analysis is developed. The time dependent, one-dimensional mass conservation equation was used in the analysis with neglecting the effects of flow and friction along the main pipe. Also isothermal gas expansion is assumed inside the main pipe and isentropic flow through the stack. The results obtained were compared very well with available data and published model.
\end{abstract}

Keywords: Gas blow down, Process safety, Pipeline rupture, Transient blow down, Transient flow

\section{Introduction}

The design of most natural gas transmission lines and distribution system is based upon steady-state calculations. Similarly the operation of the system is generally based on the same type of calculations and upon the operations experience. As a gas industry becomes more and more concerned with the identification of the reliability of a system and with optimum means of operation of a system, unsteady flow calculations are becoming more significant and necessary.

Many type of transient flow conditions are possible in a system depending mainly upon its geometry and upon the cause of the transient.

Unsteady flow (transient flow) of gas pipeline occurs during blow down or after rupture of a pipeline element. In the first case the exit cross-sectional area of the riser is much smaller than that of the main pipe. Consequently, the gas velocity in the pipeline is typically about $(10-30 \mathrm{~m} / \mathrm{s})$ during sonic discharge and the flow can be regarded as quasi-steady. Botros et al., (1989) stagnation pressure at the stack exit, which determines the discharge flux to the atmospheric, is a function of pipeline pressure at the stack entrance and pressure losses in the riser. In the case of rapture, however, the exit area can be as the main pipe cross-sectional area and thus flow velocity in the pipe becomes high (about 35\% initially at the rapture section) and varies rapidly with time (Botros et al., 1989).

Tayler et al. (1962) developed a computer program to simulate gas transmission line operating under transient condition. This program is built on the basic mass and momentum balance equation. An implicit method to calculate transient pressure and flow in gas network was presented by (Wylie et al. 1971; Rachford, 1977) presented a numerical analysis using Galerken technique to solve transient gas transportation problem through a single pipe and two pipes transmission system with compressor station modeled as separated work. Osiadacz (1982) presented a new technique to calculate the transient flow in high pressure transportation system. In this work a partial differential equation characterizing the dynamic gas flow through pipeline and a numerical scheme for its solution are considered. Rene Flatt (1985) studied unsteady frictional flow of compressible fluid generating in along pipeline after an

Accident (rupture).A high numerical accuracy obtained if the number of grid points is sufficiently large and if the special modified form of the boundary condition at the broken section is used. Botros et al. (1989) presented and discussed computational procedures entails various assumptions. Analytical and numerical solutions with effect of stack entrance and friction losses discharge coefficient are evaluating.

This paper describes a physical model of pipeline section, which is regarded as a volume with stagnation conditions inside or as a pipe with velocity increasing towards the exit. The analytical solution of mass conservation equation is given.

Estimation of gas pipeline blowdown time requires the determination of the pressure-time history for the unsteady discharge of gas through a blowdown stack open to the atmosphere. Computational procedures entail various assumptions whose validity and contributions to the accuracy are often not assessed. This paper 
discusses the computational models and solution methods and further assesses the significance of the various assumptions involved.

Volume and pipe models, numerical and analytical methods of solution, effects of stack entrance and friction losses and discharge coefficient are evaluated. The accuracy of a particular model or method of solution is greatly dependent on the $f L / D$ ratio of the pipe section under blowdown. Comparison with field measurements of a straight pipe section and a complicated compressor station yard piping enabled evaluation of the above models (K.K.K. Botros, 1989).

The unsteady frictional flow of a compressible fluid generated in a long pipeline after an accidental rupture is of considerable interest to the offshore gas industry. It answers several important questions concerning safety and pollution, e.g. the flow rate at the broken pipe end.

Laboratory tests cannot simulate the rather complex phenomenon satisfactorily. The problem is highly non-linear and no general analytical solution is yet known.

The study that based on computational fluid dynamics, the simplifying assumptions of isothermal and low Mach number flow often applied in the case of unsteady compressible flows in pipelines, have not been used.

Owing to the choking condition $(M a=1)$ which prevails for some time at the broken end. And the cumulative effect of friction over the $145 \mathrm{~km}$ long pipeline, we obtain $(\partial p / \partial x)^{t} \rightarrow-\infty$. This analytically established singularity leads to numerical difficulties which seriously affect the accuracy. For short tubes (such as shock tubes) this negative feature is much less severe. Special procedures were necessary to keep the accuracy within the chosen limit of 1 per cent (Renne, 1986).

\section{Analytical Volume Model}

This model neglects the effects of flow and friction along the main pipe and hence assumes that stagnation conditions prevail inside the whole pipe section under Blowdown. Isothermal gas expansion is assumed inside the main pipe and isentropic flow through the stack.

Mass conservation requires that is:

$$
G d t=V d p
$$

The equation of state of the form:

$$
\rho=\rho(P, T)
$$

For perfect gas and $T=$ constant, Eq. (2)yields:

$$
d \rho=\frac{d P}{R T}
$$

And thus, Eq. (1) becomes:

$$
\begin{aligned}
G R T d t & =-V d P \\
\frac{d P}{d t} & =-\frac{G R T}{V} \\
P V & =m R T
\end{aligned}
$$

From Eq.(6) and Eq.(3) becomes:

$$
\frac{d P}{d t}=-\left(\frac{G}{m}\right) P
$$

The analytical solution of Eq.(7) depends on the initial condition of the problem.

The initial condition of the problem under research given as:

At

$$
t=0, \quad P=P o
$$

Since the amount of pressure is decreasing as time increasing, hence Eq.(7) have the solution:

$$
P=\psi e^{-\left(\frac{G}{m}\right) t}
$$

By using above initial condition (i.e. Eq.(8)), the result:

$$
\psi=P o
$$

And the solution of Eq.(9) becomes: 


$$
P=P o e^{-\left(\frac{G}{m}\right) t}
$$

Or

$$
\frac{P}{P o}=e^{-\left(\frac{G}{m}\right) t}
$$

For sonic flow through valve and assuming isentropic expansion from the pipe stagnation condition, the mass flow rate given by (sallet,1984) as:

$$
\begin{gathered}
G=\left(\frac{2}{k+1}\right)^{\frac{k+1}{2(k+1)}}\left(\frac{k}{R T}\right)^{\frac{1}{2}} * P o * A_{\mathrm{thr}} * C d \\
\tau \mathrm{V}=V\left(\frac{k+1}{2}\right)^{\frac{k+1}{2(k+1)}} / c^{*}
\end{gathered}
$$

From Eq.(14):

$$
C d=V\left(\frac{k+1}{2}\right)^{\frac{k+1}{2(k+1)}} / \mathrm{c}^{*} A_{t h}
$$

Substitute Eq.(15) into Eq.(13 ) yields:

$$
G=(k / R T)^{\frac{1}{2}} *\left(\mathrm{PV} / \mathrm{c} \tau_{\mathrm{v}}\right)
$$

For perfect gas:

$$
P V=m R T
$$

Substitute Eq.(17) into Eq.(16) given:

$$
G=m(k R T)^{\frac{1}{2}} / \tau_{\mathrm{v}}
$$

Since

$$
c=(k R T)^{\frac{1}{2}}
$$

Substitute Eq.(19) into Eq.(18) yield:

$$
G=m / \tau_{\mathrm{v}}
$$

Substitute Eq.(20) into Eq.(12) yield:

$$
\frac{P}{P o}=e^{-t /} \tau_{\mathrm{v}}
$$

Or

$$
\frac{P}{P o}=e^{-\bar{t}}
$$

Where

$$
\bar{t}=\mathrm{t} / \tau_{\mathrm{v}}
$$

\section{Numerical volume solution}

The mass conservation equation can be calculated as Eq.(7):

$$
\frac{d P}{d t}=-\left(\frac{G}{m}\right) P
$$

The gas mass flow rate can be calculated using Eq.(13) as:

$$
G=\left(\frac{2}{k+1}\right)^{\frac{k+1}{2(k+1)}}\left(\frac{k}{R T}\right)^{\frac{1}{2}} * P o * A_{\mathrm{thr}} * C d
$$

and from Eq.(23) the time constant can be calculated as:

$$
\bar{t}=\mathrm{t} / \tau_{\mathrm{v}}
$$

hence,

$$
t=\bar{t} \cdot \mathrm{\tau}_{\mathrm{v}}
$$

and

$$
\overline{d t} \mathrm{v} d t=\tau
$$


Substitute Eq.(25) into Eq.(7) above yield:

$$
\frac{d P}{\overline{d t}}=-\tau v\left(\frac{G}{m}\right) P
$$

From Eq.(19), the relationship between the gas mass flow rate and time constant given as:

$$
G=\mathrm{m} / \tau_{\mathrm{v}}
$$

Substituting Eq.(19) into Eq.(26) yield:

$$
\frac{d P}{\overline{d t}}=-P
$$

Eq.(27), were solved numerically using Rung-Kutta method, by using MATLAB program (using m.file code23) to calculate the pressure ratio as function of time constant $(\bar{t})$.

\section{Results and Discussion}

An analytical and numerical solution was performed on a blowdown of straight section of pipe through one riser at the end.

A very simple relationship between dimensionless pressure and normalized time was given. The derived equation is similar to that obtained by Botros et al. (1989) [volume model analysis], so that the both results are identical in this case. From all figures the dimensionless pressure is linearly decreasing with the time. This behavior coming from the fact that the pressure at the blwdown start has been the maximum value and after short time the value of pressure was decreased and the back pressure (pressure at the downstream region) becomes affected until the sonic venting stopped.

Fig.(1) is given a comparison between the analytical and numerical results obtained from volume model and analytical results from volume model too, given by Botros et al.(1989). An identical completely behavior is obtained. Fig. (2) shows a comparison between the analytical and numerical volume model results obtained and the analytical pipe model results given by Botros et al.(1989).The volume model results(analytical and numerical) given a high value of pressure ratio than the analytical pipe model results. The reason is coming from that the volume model neglects the effects of the flow and friction along the main pipe. Hence assumes that stagnation conditions prevail inside the whole pipe section under blowdown whereas in the pipe model the effects of the flow and friction along the pipe is represents.Also, notice that the analytical and numerical solutions are no longer straight lines on the semi-log graph from the initial condition to sonic discharge. This deviation indicates to end of sonic venting through the blowdown process. This is occurs in the all figures plotted.

The numerical results were obtained by using Rung-Kutta numerical technique and by using MATHLAB computer program to calculate the dimensionless pressure drop through the blowdown process. As a result, the two methods [analytical and numerical] volume models gives only one profile of the dimensionless pressure up to the end of sonic discharge independent of the initial pressure.

Avery good agreement was obtained with analytical results model presented or with numerical results given by Botros et al. (1989).

\section{Conclusions}

Analytical and numerical verified against previous models on horizontal pipeline section enable to evaluating of validity of volume model gave the following conclusions:

1. The physical properties of the gas transporting by pipeline un affected the pressure through the blow down process.

2. The analytical and numerical volume model presented are a suitable to a simulate transient gas blow down may be consider.

3. A good agreement among the results obtained from analysis presented and the results given by (Botros et al., 1989).

\section{Nomenclature}

$\begin{array}{lc}A_{\text {th }} & \text { Pipeline cross-sectional area } \\ c & \text { Speed of sound } \\ C_{d} & \text { Stack valve discharge coefficient } \\ G & \text { Gas mass flow rate } \\ K & \text { Gas isentropic exponent }\end{array}$




$\begin{array}{ll}\mathrm{M} & \text { Mass } \\ \mathrm{P} & \text { Pressure } \\ \mathrm{P}_{\mathrm{o}} & \text { Stagnation pressure } \\ \mathrm{R} & \text { Gas constant } \\ \mathrm{T} & \text { Time } \\ \mathrm{t}^{*} & \text { Dimensionless time } \\ \mathrm{T} & \text { Temperature } \\ \mathrm{V} & \text { Volume }\end{array}$

\section{Greek Symbols}

$\begin{array}{ll}\rho & \text { Gas density } \\ \varphi & \text { Integration constant appears in eq.(9) } \\ \tau_{v} & \text { Time constant }\end{array}$

\section{References}

Botros, K.K.and Weiss, M.H. (1989). Models and methods of simulating gas pipeline Blowdown, Can.J.Chem.Eng. Vol.67, 529-539(1989). doi:10.1002/cjce.5450670402, http://dx.doi.org/10.1002/cjce.5450670402

K.K.K.Botros. (1989). Models and Methods of Simulating Gas Pipeline Blowdown, Can. J. of Chem. Eng.,V.67,P. 529-539,1989. doi:10.1002/cjce.5450670402, http://dx.doi.org/10.1002/cjce.5450670402

Osadacz. (1984). Simulation of Transient Gas Flow in Networks", Int.J.Num.Meth.Fluids, Vol.4, 13-24(1984). doi:10.1002/fld.1650040103, http://dx.doi.org/10.1002/fld.1650040103

Rachford. (1977). A fast, Highly Accurate Means of Modeling Transient Flow in Gas Pipeline System by Variational Method, Gas Tech.J.,No.13,Vol.11,101-115(1977).

Rene Flatt. (1986). Unsteady Compressible flow in long pipelines following a rapture, Int.J.Num.Meth.Fluids, Vol.6, 83-100(1986). doi:10.1002/fld.1650060204, http://dx.doi.org/10.1002/fld.1650060204

Sallet, D.W. (1984). Thermal hydraulics of valves for nuclear applications, Nucl.Sci.and Eng.Vol.88, 220-244(1984).

Tayler, T.D., Wood, N.E., Powers, J.E. (1962). A computer simulation of gas flow in long pipeline, Soc.Petr.Eng.J. Decemper, 297-302(1962).

Wylie, E.B., Mchael A. Stoner and L. Streeter. (1971). Network system transient calculation by implicit method, Soci.Petr.Eng.J. Decemper, 356-362(1971). 


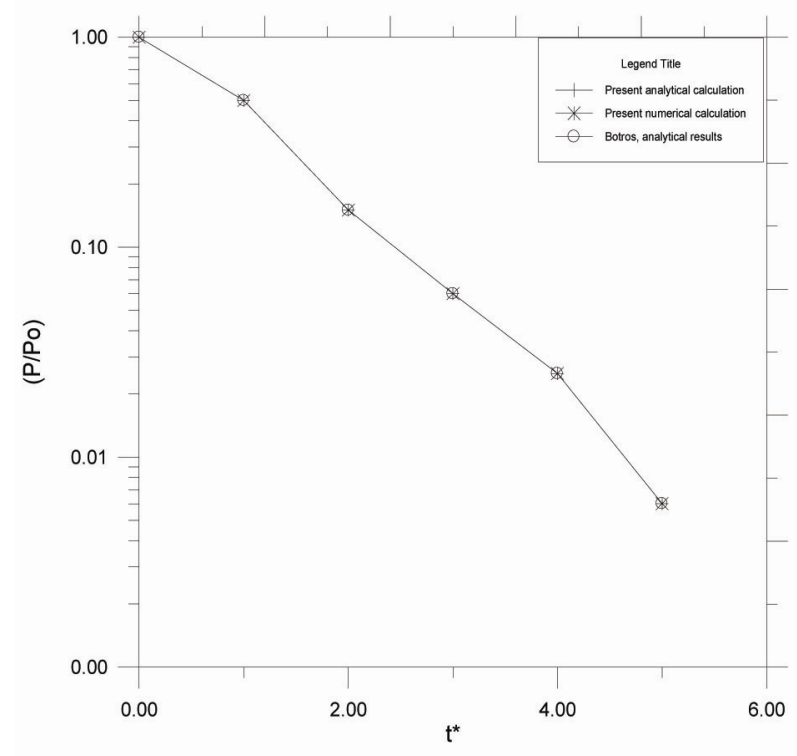

Figure 1. Pressure time profile from analytical and numerical volume models comparison with analytical volume model

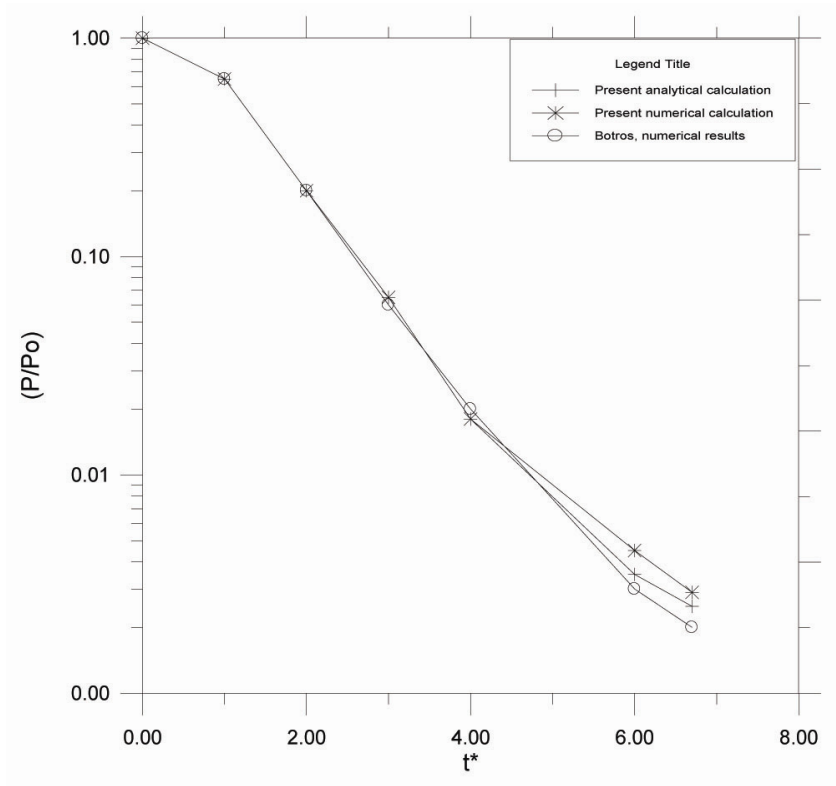

Figure 2. Pressure time profile from analytical and numerical volume models coparison with numerical pipe model results 


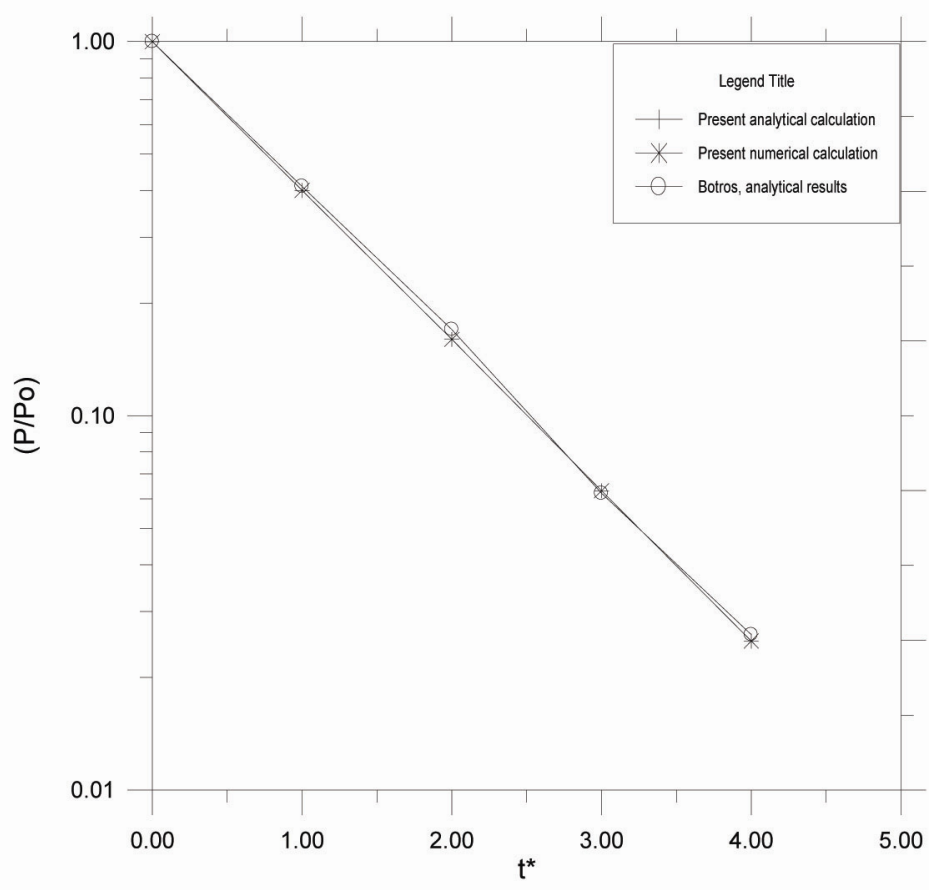

Figure 3. Pressure time profile from analytical and numerical volume models coparison with analytical pipe model

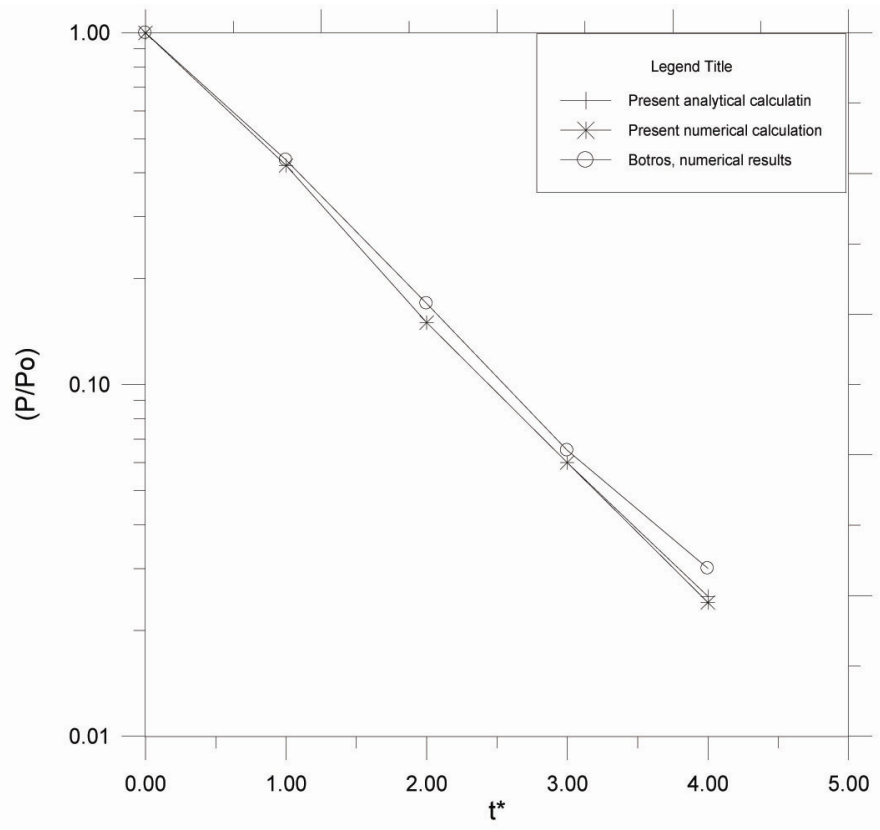

Figure 4. Pressure time profile from analytical and numerical volume models coparison with numerical pipe model 


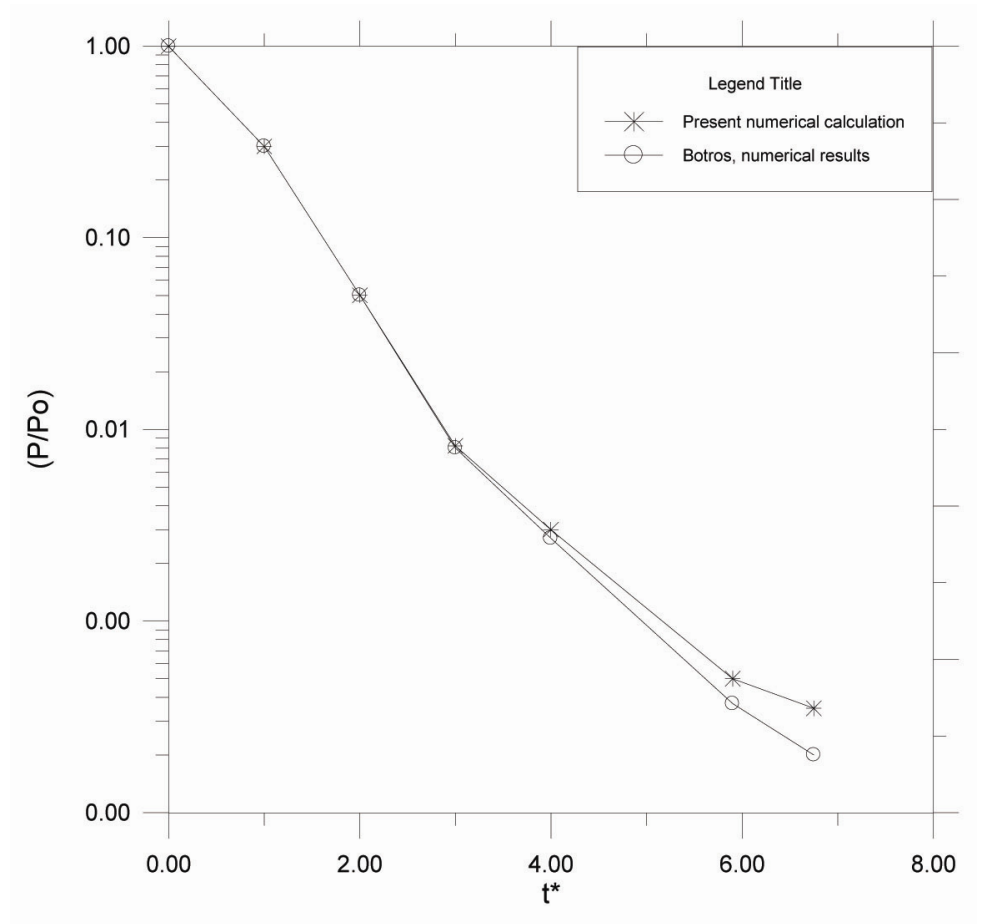

Figure 5. Pressure time profile from numerical volume model coparison with numerical pipe model

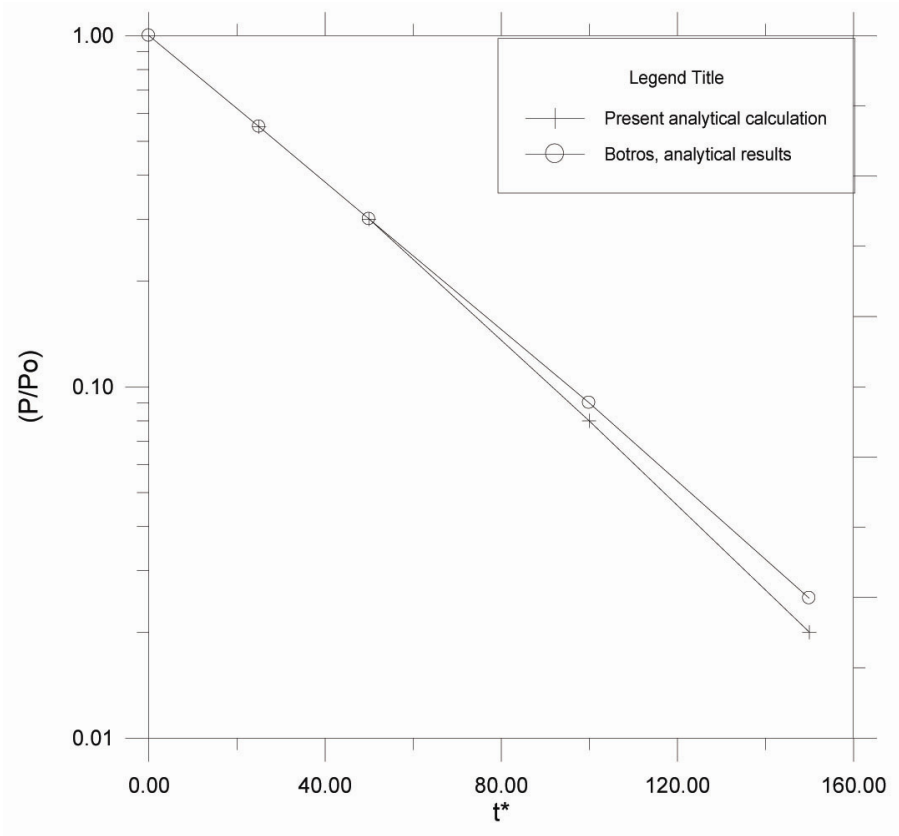

Figure 6. Pressure time profile from analytical volume model coparison with numerical pipe model 


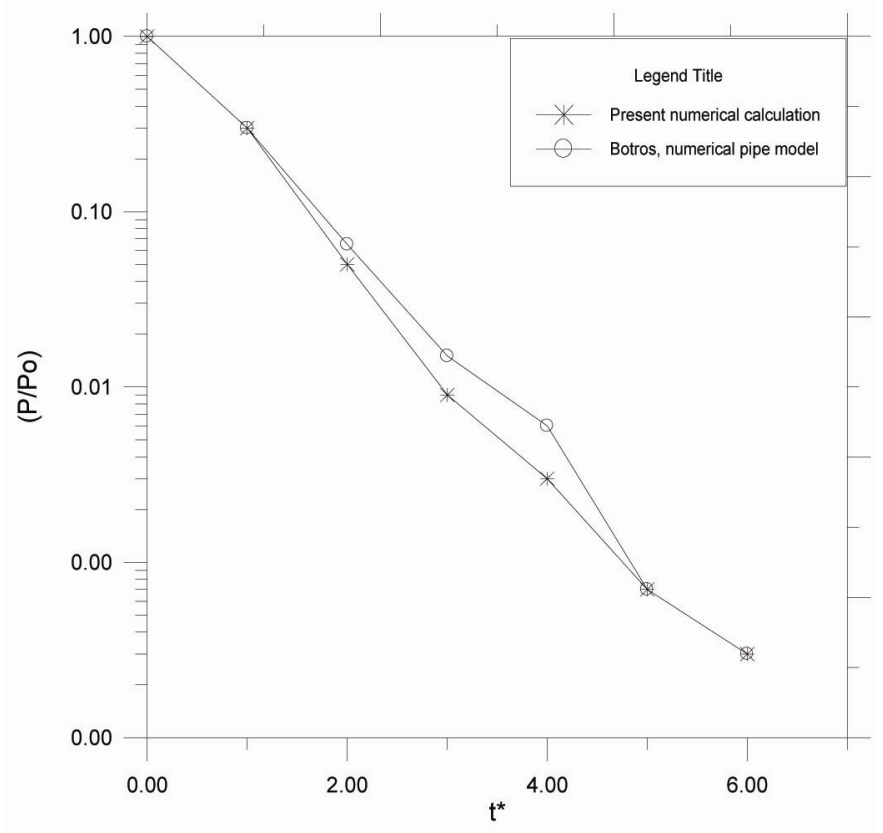

Figure 7. Pressure time profile from numerical volume model coparison with numerical pipe model 